\title{
Prognostic value of admission heart rate in patients with ST-segment elevation myocardial infarction: Role of Type 2 diabetes mellitus
}

\author{
Zhang Han, Yang Yan-min*, Zhu Jun, Liu Li-sheng, Tan Hui-qiong and Liu Yao
}

\begin{abstract}
Background: It's unknown whether the prognostic value of admission heart rate (HR) was different in patients with ST-segment elevation myocardial infarction (STEMI) with or without concomitant type 2 diabetes mellitus (T2DM).

Methods: Consecutive STEMI patients who presented within 12 hours of symptom onset were recruited from 274 hospitals in China. Participants were stratified into quartiles by admission HR. Baseline characteristics, current therapeutic recommenda- tions, laboratory biochemical tests, 30-day all-cause mortality and Cardiovascular Events (CVE, including all-cause death, reinfarction and stroke) were compared across admission HR quartiles.

Results: We evaluated 7294 STEMI patients, of these 820 (11.2\%) had known T2DM. The admission HR quartile stratification was significantly associated with all-cause mortality and CVE regardless of T2DM status $(P<0.001$ both for survival and (VE). After adjusted other risk factors, in patients without T2DM, comparing with HR $<66$ b.p.m., the increase of HR level was associated with worse prognosis $(P<0.05)$. In patients with $T 2 D M$, the hazard ratios for 30-day CVE were $1.75(95 \% \mathrm{Cl}), 1.92(95 \% \mathrm{Cl}), 3.00$ (95\%Cl) in the HR of 66-76 b.p.m., 77-88 b.p.m., and >88 b.p.m., respectively. Results were similar for 30-day all-cause mortality, but the hazard ratios in Q2 ( $P=0.139$ and $P=0.086$ for survival and CVE, respectively) and $Q 3$ groups were non-significant $(P=0.072$ and $P=0.033$ for survival and $C V E$, respectively). There was a significant interaction effect of HR and T2DM on 30-day CVE mortality $(P=0.035)$, which was not found on all-cause mortality $(P=0.126)$.
\end{abstract}

Conclusion: Admission heart rate was an important risk factor of 30-day all-cause mortality and CVE in patients with STEMI with or without T2DM. However, the predictive effect was modified by T2DM.

Keywords: Heart rate, ST-segment elevation myocardial infarction, Type 2 Diabetes Mellitus, Prognosis

\section{Introduction}

Several epidemiological and clinical studies have reported the association between heart rate (HR) and prognosis in general population and in patients with hypertension, stable coronary artery disease (CAD), heart failure (HF) [1-4], or acute coronary syndromes (ACS) [5,6].

In addition, the relationship between type 2 diabetes mellitus (T2DM) and prognosis has been well demonstrated in patients with acute myocardial infarction (AMI) $[7,8]$. Cardiac autonomic neuropathy (CAN) and higher HR may correlated with worse prognosis in diabetic patients after AMI [9,10]. However, in patients with ST-segment

\footnotetext{
* Correspondence: yymwin@yahoo.com.cn

Emergency and Intensive Care Center, Fuwai Hospital, Chinese Academy of Medical Sciences and Peking Union Medical College, Beijing, China
}

elevation myocardial infarction (STEMI), it is unclear whether the effect of HR on prognosis will be different between patients with and without T2DM.

The purpose of our report from a large study is to evaluate the association between admission HR and 30day all-cause mortality and cardiovascular events (CVE) in STEMI patients with or without T2DM.

\section{Methods \\ Patients}

From July 2001 to July 2004, 7510 consecutive patients with a clinical diagnosis of STEMI were admitted to 247 hospitals throughout China within $12 \mathrm{~h}$ of the onset of symptom. Data were collected and recorded in a research database. AMI was diagnosed on the basis of the European Society of 
Cardiology and American College of Cardiology criteria [11]. STEMI was defined as AMI with ST-segment elevation in two contiguous leads or new left-bundle branch block on an ECG obtained at the time of admission. Only patients with sinus rhythm were included in analysis. Patients who were hemodynamically unstable and hospitalized with atrial fibrillation, second or third degree heart block, ventricular tachycardia, ventricular fibrillation and cardiac arrest were excluded. According to the exclusion criteria, 99 patients were excluded. 27 patients were excluded from analysis because of incomplete information.

\section{Data collection}

At baseline, demographic characteristics and details of concomitant cardiovascular risk factors were recorded. Furthermore, a medical history was taken and fasting plasma glucose was assessed. HR was measured by a 12-lead ECG at admission. Qualifying patients received thrombolytic therapy or underwent percutanous coronary intervention (PCI) according to current therapeutic recommendations [12]. In-hospital and 30-day adverse events, including death, cardiogenic shock, HF, life-threatening arrhythmia and re-infarction were also recorded in detail.

The presence of known T2DM was recognized if the diagnosis was established according to the WHO definition [13] prior to enrollment, reported in the medical records, declared directly by the patient, or revealed by using the glucose-lowering medications, for example, metformin.

The follow-up duration was 30 days. Trained study personnel recorded all outcomes by reviewing the medical record, telephone or email contact. All-cause mortality and Cardiovascular Events (CVE, including all-cause death, reinfarction and stroke) in 30 days, were adjudicated by a central committee of clinicians.

The study was approved by the institutional Ethics committees at all participating hospitals. Informed consent was obtained from each patient.

\section{Study outcomes and definition}

The primary outcomes were death from cardiac or noncardiac cause in 30 days. CVE comprised of all-cause mortality, reinfarction, or stroke. Definitions of outcomes were as follows:

All-cause mortality included cardiovascular death (defined as any death with a cardiovascular cause including those deaths following cardiovascular procedures or surgery or deaths due to unknown cardiovascular cause) and non-cardiovascular death (defined as deaths due to a clearly documented non-cardiovascular cause).

Reinfarction was defined as the presence of at least two of the following criteria:

1. New onset of characteristic ischemic chest pain/ symptoms occurring at rest or with minimal exercise;
2. Elevation of enzyme levels or markers (CK, CK-MB, other cardiac enzymes or Troponin I or T) to at least twice the upper limit of the normal reference range or if enzymes were already elevated, greater than $50 \%$ of the lowest recovery enzyme level from the index infarction;

3. New ECG changes compatible with ischemia.

Stroke was defined as the presence of a new focal neurologic deficit consistent with a vascular origin with signs or symptoms lasting $>24$ hours. Confirmation by CT scan or MRI is was obtained when available.

\section{Statistical analysis}

Continuous variables are expressed as mean \pm standard deviation, and categorical variables as counts and percentages. Admission HR was firstly analyzed as a continuous variable, and then as a categorical variable (Q1:<66; Q2:66-76; Q3:77-88; Q4:>88b.p.m., beats per minute). Differences in baseline characteristics across admission HR quartiles were evaluated using the one-way analysis of variance (ANOVA) or Chi-square tests, according to T2DM status. The Kaplan-Meier curves were computed for cardiovascular mortality and CVE. The log- rank test was used to test the differences in the unadjusted survival curves. Hazard ratios (HRs) were estimated by the Cox proportional hazard regression model in patients with or without T2DM and the models were corrected for age, sex, systolic blood pressure(SBP), previous hypertension, myocardial infarction, heart failure (CHF), or stroke, STsegment elevation leads, killip class, and intervention measures and drugs. Potential interaction (effect modification) between HR and diabetes was evaluated by adding a multiplicative interaction term $(\mathrm{HR} \times$ diabetes $)$ to the Cox model.

A two-sided P-value of 0.05 was considered statistically significant; all analyses were performed with SPSS 17.0 for Windows (SPSS Inc., Chicago, IL, USA).

\section{Results}

Of the 7510 patients enrolled between July 2001 and July 2004 from 274 centers across China, data from 7294 patients $(97.1 \%)$ were available for analysis, and 820 (11.2\%) had known T2DM (Figure 1). Overall, median admission HR was 76 (65-88) b.p.m.; 76 (64-88) b.p.m. and 80 (68-94.5) b.p.m., respectively, in patients without and with T2DM.

\section{Baseline characteristics}

Comparison of baseline demographic and clinical characteristics in hospital between patients with and without DM is detailed in Table 1. Patients with T2DM were older $(64.7 \pm 9.9$ vs $62.3 \pm 12.0, \mathrm{P}<0.001)$ and more of them were women $(43.1 \%$ vs $27.1 \%, \mathrm{P}<0.001)$. More of the patients with T2DM were hypertensive $(57.4 \%$ vs 


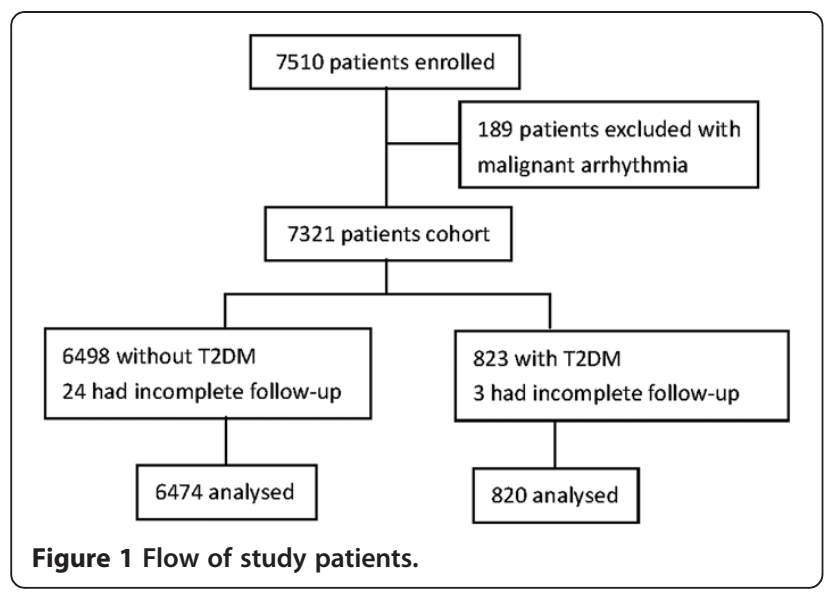

$38.5 \%, \mathrm{P}<0.001)$, had a previous stroke $(16.5 \%$ vs $8.4 \%$, $\mathrm{P}<0.001)$, HF (6.3\% vs $2.2 \%, \mathrm{P}<0.001)$ or myocardial infarction $(12.2 \%$ vs $7.5 \%, \quad \mathrm{P}<0.001)$ compared with patients without T2DM. T2DM was also associated with higher admission HR $(82.2 \pm 19.9$ vs $77.2 \pm 17.8$, $\mathrm{P}<0.001)$ and fasting serum glucose level $(13.2 \pm 5.5$ vs $7.9 \pm 3.6, \mathrm{P}<0.001)$ and more likely to be treated with insulin infusion $(40.2 \%$ vs $11.5 \%, P<0.05)$. Interestingly, participants with T2DM were more likely to

Table 1 Baseline characteristics and therapy of study participants according to diabetic state

\begin{tabular}{lccr}
\hline & $\begin{array}{c}\text { Non T2DM } \\
(\mathbf{n}=\mathbf{6 4 7 4})\end{array}$ & $\begin{array}{l}\text { T2DM } \\
(\mathbf{n}=\mathbf{8 2 0})\end{array}$ & P-value \\
\hline Females, \% & 27.1 & 43.1 & $<0.001$ \\
Age & $62.3 \pm 12.0$ & $64.7 \pm 9.9$ & $<0.001$ \\
Systolic blood pressure & $126.5 \pm 25.1$ & $128.1 \pm 26.0$ & 0.039 \\
Admission heart rate & $77.2 \pm 17.8$ & $82.2 \pm 19.9$ & $<0.001$ \\
Anterior ST Elevation, \% & 53.4 & 52.6 & 0.633 \\
Killip class II-IV, \% & 17.1 & 24.8 & $<0.001$ \\
Glucose Level, mmol/L & $7.9 \pm 3.6$ & $13.2 \pm 5.5$ & $<0.001$ \\
Previous medical history & & & \\
Hypertension, \% & 38.5 & 57.4 & $<0.001$ \\
Myocardial Infarction, \% & 7.5 & 12.2 & $<0.001$ \\
Heart failure, \% & 2.2 & 6.3 & $<0.001$ \\
Stroke, \% & 8.4 & 16.5 & $<0.001$ \\
Therapeutic measures & & & \\
PCl, \% & 10.9 & 16.9 & $<0.001$ \\
Thrombolytic therapy & 53.3 & 45.1 & $<0.001$ \\
Insulin & 11.5 & 40.2 & $<0.001$ \\
B-blockers, \% & 62.1 & 63.3 & 0.506 \\
ACEl, \% & 71.8 & 73.3 & 0.367 \\
Lipid-lowering drugs, \% & 71.1 & 72.6 & 0.377 \\
\hline PCl, percutan & & & \\
\hline
\end{tabular}

$\mathrm{PCl}$, percutaneous coronary intervention; $\mathrm{ACEl}$, Angiotensin-converting enzyme inhibitors. receive PCI $(16.9 \%$ vs $10.9 \%, \mathrm{P}<0.001)$, while fewer of them were treated with thrombolytic therapy $(45.1 \%$ vs $53.3 \%, \mathrm{P}<0.001)$.

Characteristics were reported in Table 2 by HR quartiles and by diabetic state. It's similar that previous history of stroke and serum glucose level were not associated with admission HR $(\mathrm{P}>0.05)$ in both diabetics and nondiabetics. Meanwhile, admission HR was associated with systolic blood pressure, frequency of anterior ST Elevation, Killip class II-IV, previous history of myocardial infarction or heart failure $(P<0.05)$. In addition, patients with a higher admission HR were were more likely to be treated with $\beta$-blockers, angiotensin-converting enzyme inhibitors (ACEI) and percutaneous coronary intervention (PCI) $(P<0.05)$ regardless of T2DM status.

In non-T2DM group, patients with higher admission HR were older, while more of them were women and hypertensive than those with lower admission HR $(P<0.05)$. They were more likely to use insulin, and less likely to be treated with thrombolytic therapy $(\mathrm{P}<0.05)$. However, these differences across admission HR quartiles were not apparent in patients accompanied with T2DM.

\section{Primary and secondary outcomes}

30-day outcomes were reported in Table 3 grouped by HR quartiles and by diabetic status. Patients with T2DM showed higher events rate for 30-day all-cause mortality $(9.6 \%$ vs. $15.7 \%, P<0.001)$ and CVE $(11.2 \%$ vs. $18.1 \%, P<0.001)$ compared with patients without T2DM. Events rate for 30-day all-cause mortality and CVE were higher with an increase of admission HR in patients independent of T2DM state $(P<0.001)$.

Kaplan-Meier curves for all-cause mortality and CVE were shown in Figure 2. The log-rank test reported that the unadjusted survival curves were not different among the lower three groups $(P>0.05$, multiple comparison data not shown), while patients in the highest quartile of admission HR showed the highest rate of mortality and CVE $(P<0.001)$, regardless of T2DM.

Multivariable Cox regression analysis was performed to assess the prognostic value of admission HR after adjusting for age, sex, the medical history of hypertension, heart failure, myocardial infarction, stroke, Killip class, medications and procedures in-hospital (Insulin, $\beta$-blockers, ACEI, lipid-lowering drugs, and PCI). Analyses with HR as a continuous variable showed that admission HR was an independent risk factor of 30-day all-cause mortality (HR, 1.011; 95\% CI, 1.008-1.015, $P<0.001)$ and CVE (HR, 1.010; 95\% CI, 1.006-1.013, $P<0.001)$ in overall patients and in patients without T2DM (for all-cause mortality: HR, 1.013; 95\% CI, 1.009-1.017, $P<0.001$; for CVE: HR, 1.010; 95\% CI, 1.006-1.014, $P<0.001)$. The similar predictive effect were found for 30-day CVE $(\mathrm{P}<0.05)$. 
Table 2 Baseline characteristics and therapy of non-diabetic patients based on quartiles of admission heart rate (Q1-Q4)

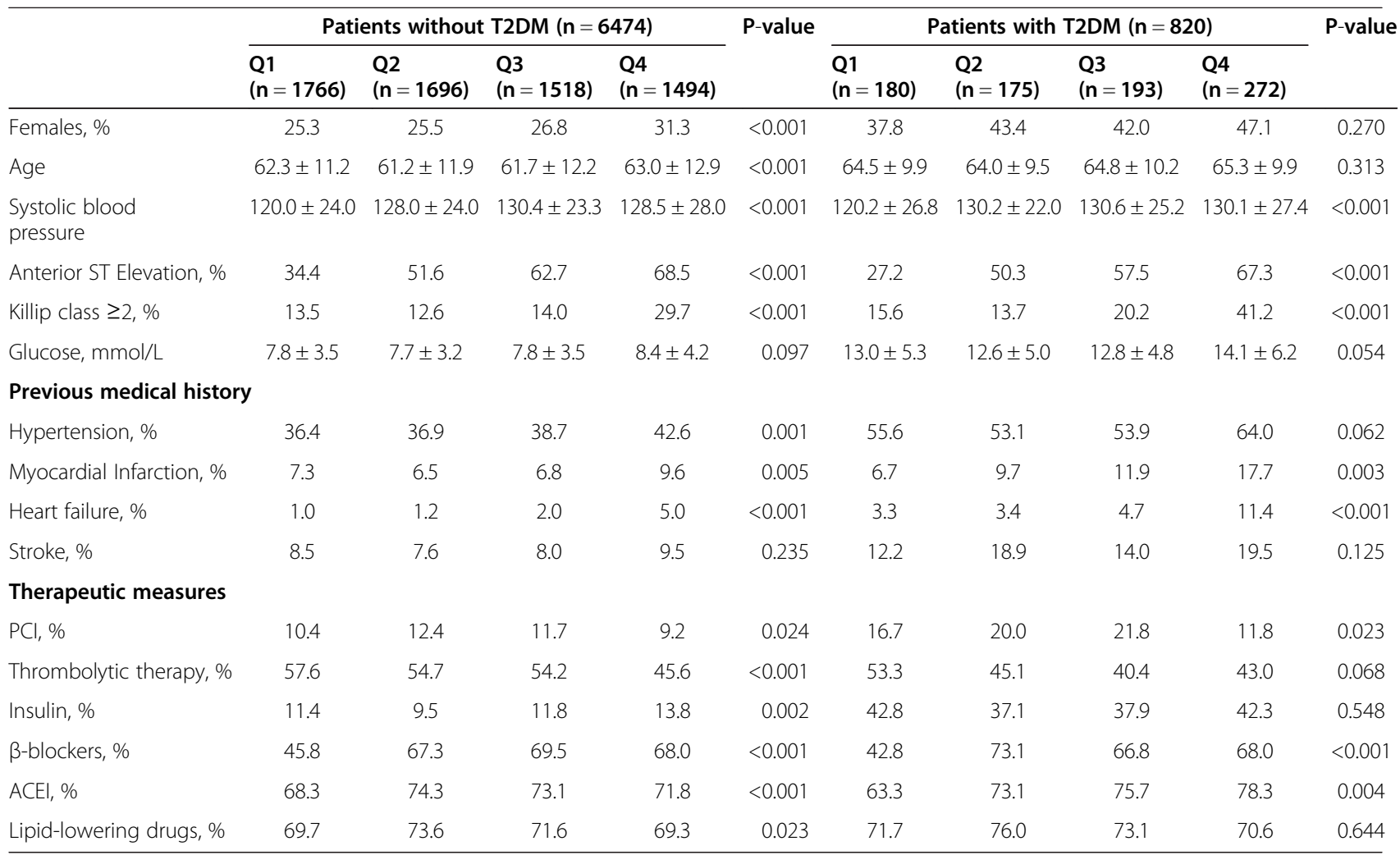

$\mathrm{PCl}$, Percutaneous coronary intervention; ACEl, Angiotensin-converting enzyme inhibitors; P-values are given for the comparison of between the different quartiles of heart rate.

Table 4 shows the details of association between quartiles of admission $\mathrm{HR}$ and prognosis. In patients without T2DM, comparing with HR <66 b.p.m., the HR of $66-76$ b.p.m., 77-88 b.p.m., or >88 b.p.m. demonstrate Hazard Ratios of 1.44 (95\% CI 1.13-1.84, P = 0.003), 1.41 (95\% CI $1.10-1.80, \mathrm{P}=0.007$ ), and 2.30 (95\% CI 1.85-2.87, $\mathrm{P}<0.001$ ) for 30-day all-cause mortality; while hazard ratios for 30day CVE were 1.39 (95\% CI, 1.10-1.75, P = 0.005), 1.42 (95\% CI 1.22-1.78, $\mathrm{P}=0.003$ ) and 2.30 (95\% CI 1.87-2.83, $\mathrm{P}<0.001)$. And in patients with T2DM, the hazard ratios for 30-day CVE were 1.75(95\%CI), 1.92(95\%CI), 3.00

Table 3 30-day events stratified by heart rate quartiles (Q1-Q4) in patients with and without T2DM

\begin{tabular}{llllllll}
\hline Variable & \multicolumn{5}{c}{ Heart rate(b.p.m.) } & P-value \\
\cline { 2 - 6 } & All & Q1 & Q2 & Q3 & Q4 & \\
\hline Patients without T2DM & & & & & & \\
All-cause mortality (\%) & 9.6 & 6.9 & 7.0 & 8.0 & 17.3 & $<0.001$ \\
Cardiovascular events (\%) & 11.2 & 8.8 & 8.8 & 9.6 & 18.5 & $<0.001$ \\
Patients with T2DM & & & & & & \\
All-cause mortality (\%) & 15.7 & 9.4 & 11.4 & 13.0 & 24.6 & $<0.001$ \\
Cardiovascular events (\%) & 18.1 & 10.6 & 12.6 & 16.0 & 27.9 & $<0.001$ \\
\hline
\end{tabular}

(95\%CI) in the HR of 66-76 b.p.m., 77-88 b.p.m., and >88 b.p.m., respectively. Similar results were shown in the analyses of 30-day all-cause mortality (As is shown in Table 4), although the hazard ratios in Q2 and Q3 groups were non-significant, which seemed probably due to inefficient power with the relative small sample size in patients with T2DM.

However, of note, there was a significant interaction effect of HR and diabetic state on 30-day CVE mortality $(P=0.035)$, which indicated that increased heart rate at any level is more deleterious for CVE in diabetic than non-diabetic individuals. The potential interaction effect on all-cause mortality was not found $(\mathrm{P}=0.126)$.

\section{Discussion}

Our study is the first one to assess the association between admission $\mathrm{HR}$ and all-mortality and CVE in STEMI patients when accompanied with or without T2DM. The main results are summarized as follows:

1. In STEMI patients accompanied with T2DM, admission HR, 30-day all-cause mortality and rate of CVE was higher compared with those without T2DM. 


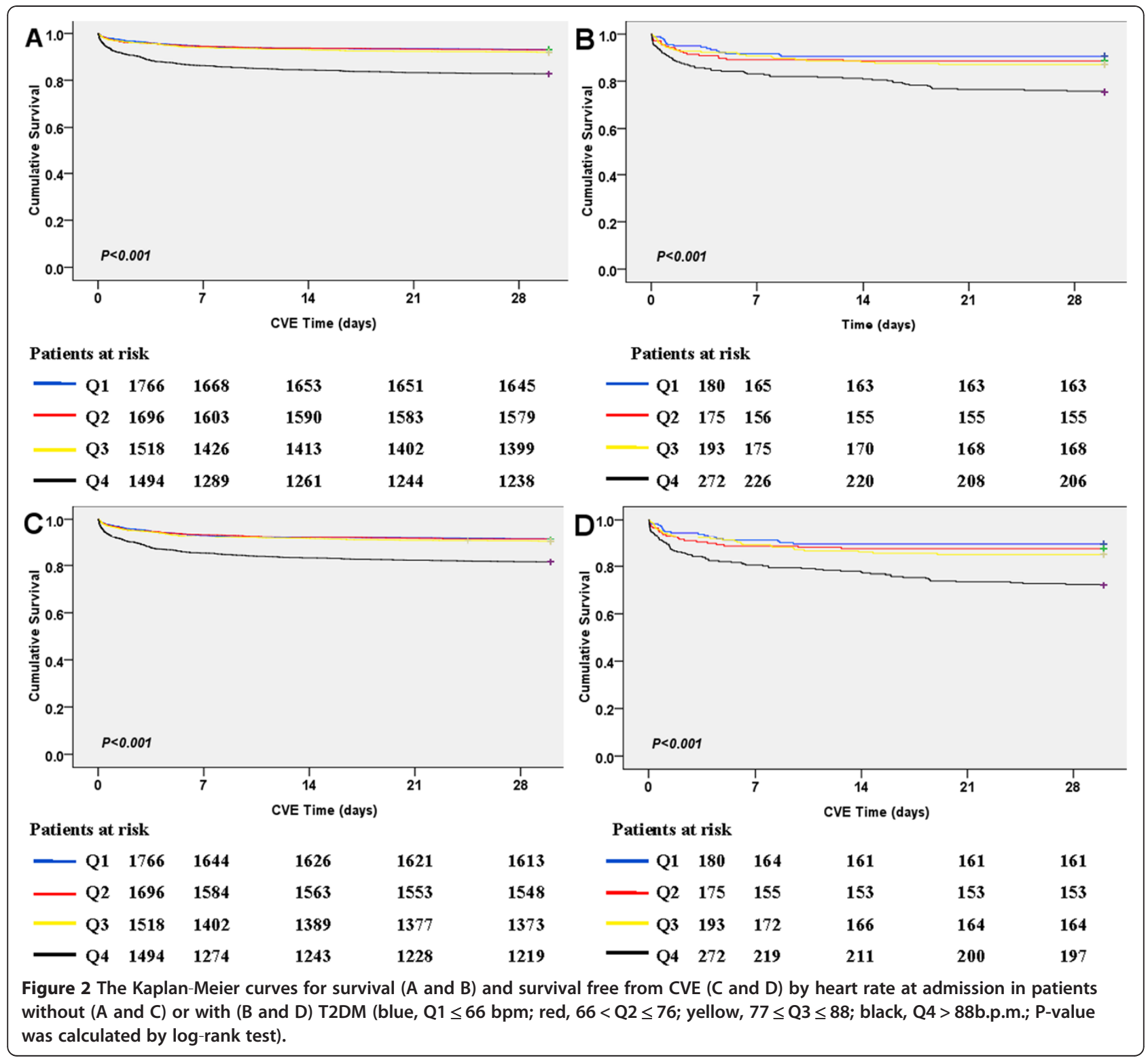

2. Admission HR was an independent risk factor of prognosis in STEMI patients with or without T2DM.

3. After controlling for baseline and in-hospital therapeutic confounders, the prognostic effect of HR was different between patients with T2DM and without T2DM. In the T2DM patients, the hazard ratios in Q2 and Q3 groups were non- significant, however, it seemed probably due to inefficient power

Table 4 Adjusted hazard ratios for heart rate at admission in patients with or without T2DM

\begin{tabular}{|c|c|c|c|c|c|c|c|}
\hline & \multicolumn{7}{|c|}{ Admission heart rate(b.p.m.) } \\
\hline & $\overline{Q 1}$ & Q2, $\mathrm{HR}(95 \% \mathrm{Cl})$ & P-value & Q3, HR(95\%Cl) & P-value & Q4, HR(95\%Cl) & P-value \\
\hline \multicolumn{8}{|l|}{ Patients without T2DM } \\
\hline All-cause mortality & 1.00 & $1.44(1.13-1.84)$ & 0.003 & $1.41(1.10-1.80)$ & 0.007 & $2.30(1.85-2.87)$ & $<0.001$ \\
\hline Cardiovascular events & 1.00 & $1.39(1.10-1.75)$ & 0.005 & $1.42(1.22-1.78)$ & 0.003 & $2.30(1.87-2.83)$ & $<0.001$ \\
\hline \multicolumn{8}{|l|}{ Patients with T2DM } \\
\hline All-cause mortality & 1.00 & $1.70(0.84-3.41)$ & 0.139 & $1.84(0.95-3.59)$ & 0.072 & $2.56(1.38-4.73)$ & 0.003 \\
\hline Cardiovascular events & 1.00 & $1.75(0.92-3.33)$ & 0.086 & $1.92(1.05-3.48)$ & 0.033 & $3.00(1.76-5.14)$ & $<0.001$ \\
\hline
\end{tabular}


with the relative small sample size. Hazard ratios in each increased HR group were larger in individuals with than without T2DM, and there was a significant interaction effect of $\mathrm{HR}$ and diabetic state on 30-day CVE mortality $(\mathrm{P}=0.035)$, which indicated that increased heart rate at any level is more deleterious for prognosis in diabetic than non-diabetic individuals.

Previous studies have shown a positive association between elevated $\mathrm{HR}$ and increasing risk of cardiovascular disease (CVD), all-cause and cardiovascular mortality in general people and in patients with various cardiovascular diseases [1,3,14-16], especially AMI. Myocardial ischemia results from an imbalance between coronary blood flow supply and myocardial metabolic demand. HR and other determinants highly affect myocardial oxygen demand [17], meanwhile, HR is the major determinant of the supply of blood flow. Some studies [18] have shown that elevated HR can stimulate the arterial wall, affect local hemodynamic environment $[19,20]$ activate inflammation [21], disturb the imbalance between myocardial demand and supply [22], and disrupt atherosclerotic plaques [23].

Concomitant T2DM then increases the aforementioned influence further when compared with patients without T2DM, propensity to worse prognosis. First, hyperglycemia may play an important role by several pathophysiological mechanisms [24-27]. Secondly, these patients have higher HR [28].

Some epidemiologic studies suggest the relationship between HR and outcome in diabetic patients. Stettler et al. [29] have reported the prognostic role of HR in patients with T2DM. In the Bremen Diabetes study, higher HR was also related to an increase of cardiovascular death [30]. However, Anselmino et al. [31] reported that in patients with stable CAD, the association between resting $\mathrm{HR}$ and CVE can be found in those with diabetes, but not in nondiabetic patients. It's unknown if the difference retains in acute settings, such as STEMI. Our observation performed after separating T2DM from non-T2DM in STEMI patients, which showed that HR was an independent risk factor of prognosis $(\mathrm{P}<0.05)$ regardless of T2DM.

Cardiac autonomic neuropathy (CAN) is a common chronic complication of T2DM [32], and manifests an increase in HR and a reduction in HR variability, that confer higher morbidity and mortality to diabetic patients $[33,34]$. CAN may contribute to the differences identified in the present study. High HR is not considered to diagnose CAN by itself, but it can reflect a relative imbalance in the sympathetic activity and vagal impairment [32]. It appears that $\mathrm{T} 2 \mathrm{DM}$ and autonomic dysfunction are causally related, and a higher HR, elevated high blood pressure, and ischemia burden may be intermediate accelerators [35]. So that, T2DM may strengthen the predictive effect of HR by CAN, but we also need further study of the internal mechanism to confirm this hypothesis.

Our finding in more than 7000 patients pointed out that HR at admission was an independent predictor of short-term adverse outcome, including cardiovascular and all-cause mortality despite the high level of treatment with beta-blockers. The results emphasized the different of prognostic effect of HR between the two kinds of population, meanwhile. However, more prospective studies are needed to confirm these findings in larger clinical registries.

\section{Study limitation}

First, the study is a subgroup analysis of an existing material, and thereby suffers from limitations and biases of such material. It is not totally equivalent to the real settings. Second, we only observed the all-cause mortality and CVE in 30 days; a longer follow-up may declare more information and elucidate the hypothesis generated in this report. Third, we don't take blood glucose or glycolated hemoglobin level into account, which reflect the therapeutic effect of T2DM, and may effect the degree of CAN. And the HR in our database is only gained at admission rather than on-treatment or discharge value simultaneously. At last, the sample size of T2DM patients was far less than patients without T2DM ( 1/8), and seemed to preclude statistical significance. A larger sample will be needed to improve the statistical power and find out the truth.

\section{Conclusion}

In conclusion, resting $\mathrm{HR}$ affects 30-day all-cause mortality and CVE in STEMI patients no matter with or without T2DM. The adverse effect of HR is more obvious in diabetic than non-diabetic individuals. When researching HR and cardiovascular prognosis, T2DM status must be considered. This is also useful in clinical study and treatment.

\section{Competing interests}

The authors declare that they have no competing interest.

\section{Authors' contributions}

$\mathrm{LL}$ and $\mathrm{ZJ}$ conceived the origninal idea and designed the research. ZH, $\mathrm{LY}$ and TH performed experiments and analyzed results. $Z \mathrm{H}$ and $Y Y$ wrote the manuscript. All authors read and approved the final manuscript.

Received: 14 April 2012 Accepted: 4 November 2012

Published: 15 November 2012

\section{References}

1. Saraiva F, Antonio N, Lourenco C, Goncalves F, Monteiro P, Goncalves L, Freitas $M$, Providencia LA: Heart rate and prognosis in acute coronary syndromes. Rev Port Cardiol 2010, 29(7-8):1101-1119. 
2. Mao Q, Huang JF, Lu X, Wu X, Chen J, Cao J, Li J, Gu D: Heart rate influence on incidence of cardiovascular disease among adults in China. Int J Epidemiol 2010, 39(6):1638-1646.

3. Palatini $P$, Benetos A, Julius S: Impact of increased heart rate on clinical outcomes in hypertension: implications for antihypertensive drug therapy. Drugs 2006, 66(2):133-144.

4. Bohm M, Swedberg K, Komajda M, Borer JS, Ford I, Dubost-Brama A, Lerebours G, Tavazzi L: Heart rate as a risk factor in chronic heart failure (SHIFT): the association between heart rate and outcomes in a randomised placebo-controlled trial. Lancet 2010, 376(9744):886-894

5. Bangalore S, Messerli FH, Ou FS, Tamis-Holland J, Palazzo A, Roe MT, Hong MK, Peterson ED: The association of admission heart rate and in-hospital cardiovascular events in patients with non-ST-segment elevation acute coronary syndromes: results from 135164 patients in the CRUSADE quality improvement initiative. Eur Heart J 2010, 31(5):552-560.

6. Honda T, Kanazawa H, Koga H, Miyao Y, Fujimoto K: Heart rate on admission is an independent risk factor for poor cardiac function and inhospital death after acute myocardial infarction. J Cardiol 2010, 56(2):197-203.

7. Mak KH, Moliterno DJ, Granger CB, Miller DP, White HD, Wilcox RG, Califf $\mathrm{RM}$, Topol EJ: Influence of diabetes mellitus on clinical outcome in the thrombolytic era of acute myocardial infarction, GUSTO-I Investigators. Global Utilization of Streptokinase and Tissue Plasminogen Activator for Occluded Coronary Arteries. J Am Coll Cardiol 1997, 30(1):171-179.

8. van der Schaaf RJ, Henriques JP, Wiersma JJ, Koch KT, Baan J Jr, Mulder KJ, Durrer JD, Tijssen JG, Piek JJ, de Winter RJ: Primary percutaneous coronary intervention for patients with acute ST elevation myocardial infarction with and without diabetes mellitus. Heart 2006, 92(1):117-118.

9. Whang W, Bigger JT Jr: Comparison of the prognostic value of RR-interval variability after acute myocardial infarction in patients with versus those without diabetes mellitus. Am J Cardiol 2003, 92(3):247-251.

10. Otter W, Kleybrink S, Doering W, Standl E, Schnell O: Hospital outcome of acute myocardial infarction in patients with and without diabetes mellitus. Diabet Med 2004, 21(2):183-187.

11. Alpert JS, Thygesen K: Myocardial infarction redefined-A consensus document of The Joint European Society of Cardiology/American College of Cardiology Committee for the Redefinition of Myocardial Infarction. Eur Heart J 2000, 21(18):1502-1513.

12. Ergelen $M$, Uyarel $H$, Cicek $G$, Isik T, Osmonov D, Gunaydin ZY, Bozbay M, Turer A, Gul M, Abanonu GB, et al: Which is worst in patients undergoing primary angioplasty for acute myocardial infarction? Hyperglycaemia? Diabetes mellitus? Or both? Acta Cardiol 2010, 65(4):415-423.

13. Report of WHO Consultation: Definition, Diagnosis and Classification of Diabetes Mellitus and its Complications. Part 1: Diagnosis and Classification of Diabetes Mellitus. Geneva: World Health Organization, Department of Noncommunicable Disease Surveillance; 1999.

14. Palatini P: Elevated heart rate: a "new" cardiovascular risk factor? Prog Cardiovasc Dis 2009, 52(1):1-5.

15. Kristal-Boneh $\mathrm{E}$, Silber $\mathrm{H}$, Harari G, Froom P: The association of resting heart rate with cardiovascular, cancer and all-cause mortality. Eight year follow-up of 3527 male Israeli employees (the CORDIS Study). Eur Heart J 2000, 21(2):116-124

16. Fosbol EL, Seibaek M, Brendorp B, Moller DV, Thune JJ, Gislason GH, TorpPedersen C, Kober L: Long-term prognostic importance of resting heart rate in patients with left ventricular dysfunction in connection with either heart failure or myocardial infarction: the DIAMOND study. Int J Cardiol 2010, 140(3):279-286

17. Tanaka N, Nozawa T, Yasumura Y, Futaki S, Hiramori K, Suga H: Heart-rateproportional oxygen consumption for constant cardiac work in dog heart. Jpn J Physiol 1990, 40(4):503-521.

18. Kaplan JR, Manuck SB, Clarkson TB: The influence of heart rate on coronary artery atherosclerosis. J Cardiovasc Pharmacol 1987 10(Suppl 2):S100-S102. discussion S103.

19. Giannoglou GD, Chatzizisis YS, Zamboulis C, Parcharidis GE, Mikhailidis DP Louridas GE: Elevated heart rate and atherosclerosis: an overview of the pathogenetic mechanisms. Int J Cardio/ 2008, 126(3):302-312.

20. Lang CC, Gupta S, Kalra P, Keavney B, Menown I, Morley C, Padmanabhan S: Elevated heart rate and cardiovascular outcomes in patients with coronary artery disease: clinical evidence and pathophysiological mechanisms. Atherosclerosis 2010, 212(1):1-8.
21. Rogowski O, Shapira I, Shirom A, Melamed S, Toker S, Berliner S: Heart rate and microinflammation in men: a relevant atherothrombotic link. Heart 2007, 93(8):940-944.

22. Orso F, Baldasseroni S, Maggioni AP: Heart rate in coronary syndromes and heart failure. Prog Cardiovasc Dis 2009, 52(1):38-45.

23. Heidland UE, Strauer BE: Left ventricular muscle mass and elevated heart rate are associated with coronary plaque disruption. Circulation 2001, 104(13):1477-1482.

24. Boudina S, Abel ED: Diabetic cardiomyopathy, causes and effects. Rev Endocr Metab Disord 2010, 11(1):31-39.

25. Creager MA, Luscher TF, Cosentino F, Beckman JA: Diabetes and vascular disease: pathophysiology, clinical consequences, and medical therapy: Part I. Circulation 2003, 108(12):1527-1532.

26. Luscher TF, Creager MA, Beckman JA, Cosentino F: Diabetes and vascular disease: pathophysiology, clinical consequences, and medical therapy: Part II. Circulation 2003, 108(13):1655-1661.

27. Hofsten DE, Logstrup BB, Moller JE, Pellikka PA, Egstrup K: Abnormal glucose metabolism in acute myocardial infarction: influence on left ventricular function and prognosis. JACC Cardiovasc Imaging 2009, 2(5):592-599.

28. Foo K, Sekhri N, Knight C, Deaner A, Cooper J, Ranjadayalan K, Suliman A, Timmis AD: The effect of diabetes on heart rate and other determinants of myocardial oxygen demand in acute coronary syndromes. Diabet Med 2004, 21(9):1025-1031.

29. Stettler C, Bearth A, Allemann S, Zwahlen M, Zanchin L, Deplazes M, Christ ER, Teuscher A, Diem P: QTc interval and resting heart rate as long-term predictors of mortality in type 1 and type 2 diabetes mellitus: a 23-year follow-up. Diabetologia 2007, 50(1):186-194.

30. Linnemann B, Janka HU: Prolonged QTc interval and elevated heart rate identify the type 2 diabetic patient at high risk for cardiovascular death. The Bremen Diabetes Study. Exp Clin Endocrinol Diabetes 2003, 111(4):215-222.

31. Anselmino M, Ohrvik J, Ryden L: Resting heart rate in patients with stable coronary artery disease and diabetes: a report from the euro heart survey on diabetes and the heart. Eur Heart J 2010, 31(24):3040-3045.

32. Pop-Busui R: Cardiac autonomic neuropathy in diabetes: a clinical perspective. Diabetes Care 2010, 33(2):434-441.

33. Maser RE, Mitchell BD, Vinik Al, Freeman R: The association between cardiovascular autonomic neuropathy and mortality in individuals with diabetes: a meta-analysis. Diabetes Care 2003, 26(6):1895-1901.

34. Buccelletti E, Gilardi E, Scaini E, Galiuto L, Persiani R, Biondi A, Basile F, Silveri NG: Heart rate variability and myocardial infarction: systematic literature review and metanalysis. Eur Rev Med Pharmacol Sci 2009, 13(4):299-307.

35. Nagaya T, Yoshida H, Takahashi H, Kawai M: Resting heart rate and blood pressure, independent of each other, proportionally raise the risk for type-2 diabetes mellitus. Int J Epidemiol 2010, 39(1):215-222.

doi:10.1186/1471-2261-12-104

Cite this article as: Han et al:: Prognostic value of admission heart rate in patients with ST-segment elevation myocardial infarction: Role of Type 2 diabetes mellitus. BMC Cardiovascular Disorders 2012 12:104.

\section{Submit your next manuscript to BioMed Central and take full advantage of:}

- Convenient online submission

- Thorough peer review

- No space constraints or color figure charges

- Immediate publication on acceptance

- Inclusion in PubMed, CAS, Scopus and Google Scholar

- Research which is freely available for redistribution 